\title{
A Conditional Random Field Weakly Supervised Segmentation Approach for Segmenting keratocytes Cells in Corneal Optical Coherence Tomography Images
}

Ameneh Boroomand

Alexander Wong

Kostadinka Bizheva
University of Waterloo., ON, Canada

University of Waterloo, ON, Canada

University of Waterloo, ON, Canada

\section{Abstract}

Keratocytes are vital for maintaining the overall health of human cornea as they preserve the corneal transparency and help in healing corneal injuries. Manual segmentation of keratocytes is challenging, time consuming and also needs an expert. Here, we propose a novel semi-automatic segmentation framework, called Conditional Random Field Weakly Supervised Segmentation (CRF-WSS) to perform the keratocytes cell segmentation. The proposed framework exploits the concept of dictionary learning in a sparse model along with the Conditional Random Field (CRF) modeling to segment keratocytes cells in Ultra High Resolution Optical Coherence Tomography (UHR-OCT) images of human cornea. The results show higher accuracy for the proposed CRF-WSS framework compare to the other tested Supervised Segmentation (SS) and Weakly Supervised Segmentation (WSS) methods.

\section{Introduction}

Keratocytes segmentation is needed to calculate keratocytes cells density that can be used as a biomarker in diagnosis and monitoring of different corneal diseases [1]. Manual segmentation of each individual keratocyte is tedious and time consuming due to the high number of these cells in the cornea, the existence of speckle noise and also the limited resolution of OCT images. In previous work, Keratocytes cells segmentation using UHR-OCT images was done using the second-order moment analysis along with the simple thresholding [1]. The sensitivity of this method to the speckle noise and different OCT artifacts makes some challenges for the keratocytes cells segmentation. Here, we propose a novel CRFWSS algorithm that is able to detect corneal keratocytes cells in a semi-automatic manner and with a limited amount of expert intervention while it has more stability against the speckle noise and OCT artifacts.

\section{Method}

In this paper, we design a novel CRF-WSS segmentation framework that takes advantage of the dictionary learning concept along with the Conditional Random Field (CRF) modeling to perform keratocytes segmentation as a weakly supervised binary classification task (Fig. 1). In the designed framework, we learn a representative dictionary, $D$, for each class of keratocytes and background using an unsupervised clustering based dictionary learning method with the following optimization,

$$
\underset{\mathbf{D}}{\arg \min } \sum_{s=1}^{K} \sum_{t=1}^{n_{s}}\left\|\mathbf{y}_{t}^{(s)}-\mathbf{d}_{s}\right\|_{2}^{2}
$$

Here, $\mathbf{y}_{t}^{(s)}$ represents the OCT data and $\mathbf{d}_{s}$ denotes to the dictionary atoms. Running this step partitions the whole OCT image to a set of bags, each includes a number of pixels and summarized by one atom from the learned dictionary $D$. We design a Multiple Instance Learning (MIL) approach [2] to train a bag-level classifier which finds weak labels for all unlabeled instances in partially labeled bags. We use a naive Bayes classifier with the weakly labeled instances to obtain an initial segmentation map for the keratocytes cells. Our proposed CRF-WSS framework also employs a sepcific designed CRF model to take account for the spatially local interactions that exist among the OCT data in to the segmentation process. Incorporating the local OCT information improves the performance of our proposed segmentation framework in cases when the OCT data has some speckle noise and artifacts.

\section{Results}

The proposed segmentation framework was tested on a sample of corneal image squired using a UHR-SD-OCT system operating at $800 \mathrm{~nm}$ spectral range. The result of applying our proposed CRFWSS framework is shown in Fig.2 (e) and compared to the other tested SS and W-SS (Fig. 2 (c,d)). Visual assessment of the results indicates the effectiveness of the proposed CRF-WSS framework in better extraction of keratocytes cells when the OCT data is noisy. For the quantitative analysis, each tested SS, WSS and CRF-WSS algorithms was run 15 times and with different number of initially labeled pixels and the accuracy curves are calculated as,

$$
\text { Accuracy }=\frac{T P+T N}{T P+F P+T N+F N} \text {. }
$$

Comparing of the calculated accuracy curves in Fig. 2(f) demonstrates that the proposed CRF-WSS framework is able to resolve keratocytes cells with higher accuracy compare to the other tested SS and WSS methods and with the different number of initially labeled pixels.

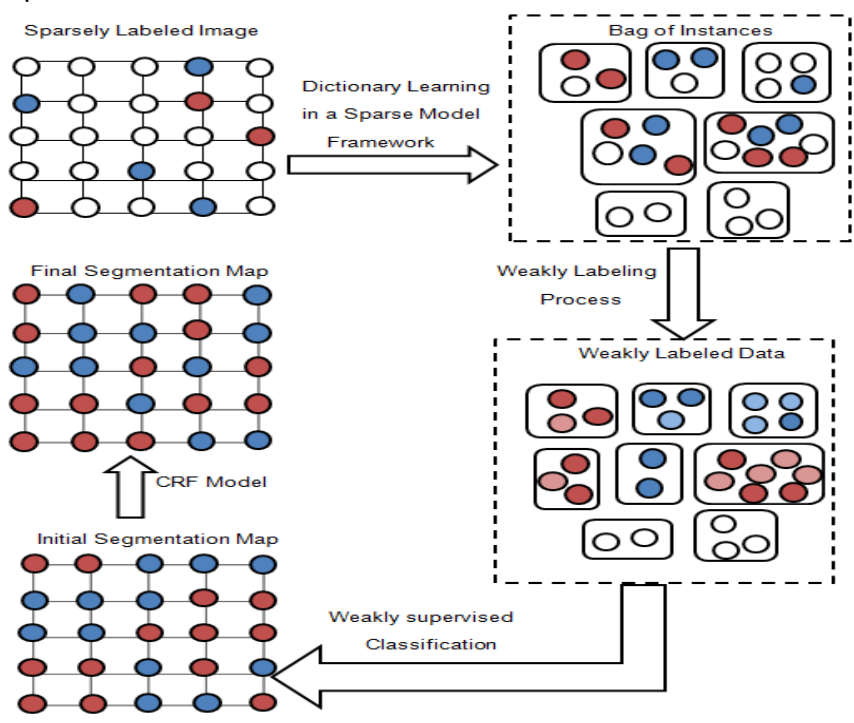

Fig. 1: Proposed CRF-WSS framework.

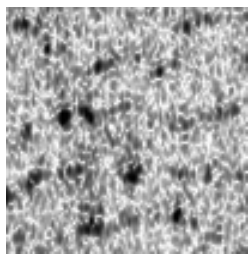

(a) UHR-OCT

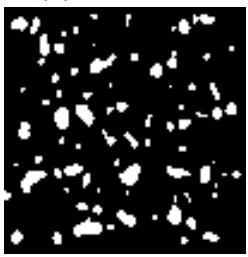

(d) WSS

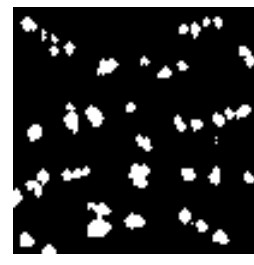

(b) Ground truth

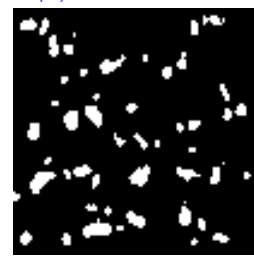

(e) CRF-WSS

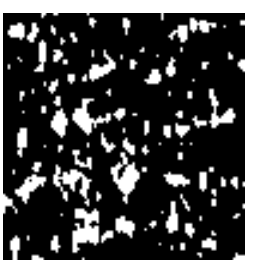

(c) SS

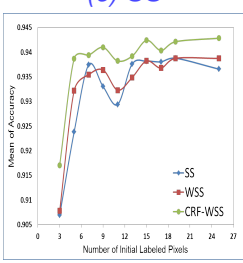

(f) Accuracy Curves
Fig. 2: (a) UHR-OCT image. (b) Ground truth. (c-e) SS, WSS and CRF-WSS results, number of initial labeled pixels=3, dictionary atoms $=5$. (f) Mean of accuracy per 15 runs of SS, W-SS and CRFWSS segmentation algorithms.

\section{References}

[1] Karimi, A.H., Wong, A. and Bizheva, K. Automated detection and cell density assessment of keratocytes in the human corneal stroma from ultrahigh resolution optical coherence tomograms. Biomedical optics express(2011).

[2] Vezhnevets, A. and Buhmann, J.M. Towards weakly supervised semantic segmentation by means of multiple instance and multitask learning.CVPR(2010). 